\title{
Coherent optical transition radiation and self-amplified spontaneous emission generated by chicane-compressed electron beams
}

\author{
A. H. Lumpkin \\ Fermilab, Batavia, Illinois 60510, USA \\ R. J. Dejus and N. S. Sereno \\ Argonne National Laboratory, Argonne, Illinois 60439, USA
}

(Received 22 December 2008; published 29 April 2009; corrected 19 May 2009)

\begin{abstract}
Observations of strongly enhanced optical transition radiation (OTR) following significant bunch compression of photoinjector beams by a chicane have been reported during the commissioning of the Linac Coherent Light Source accelerator and recently at the Advanced Photon Source (APS) linac. These localized transverse spatial features involve signal enhancements of nearly a factor of 10 and 100 in the APS case at the $150-\mathrm{MeV}$ and $375-\mathrm{MeV}$ OTR stations, respectively. They are consistent with a coherent process seeded by noise and may be evidence of a longitudinal space charge microbunching instability which leads to coherent OTR emissions. Additionally, we suggest that localized transverse structure in the previous self-amplified spontaneous emission (SASE) free-electron laser (FEL) data at APS in the visible regime as reported at FEL02 may be attributed to such beam structure entering the FEL undulators and inducing the SASE startup at those "prebunched" structures. Separate beam structures 120 microns apart in $x$ and $2.9 \mathrm{~nm}$ apart in wavelength were reported. The details of these observations and operational parameters will be presented.
\end{abstract}

DOI: 10.1103/PhysRevSTAB.12.040704

PACS numbers: 41.60.Ap, 41.60.Cr

\section{INTRODUCTION}

Several years ago, the self-amplified spontaneous emission (SASE) free-electron laser (FEL) experiments [1] at the Advanced Photon Source (APS) included a series of observations on $z$-dependent microbunching as measured by visible light coherent optical transition radiation (COTR) [2-4]. This SASE-induced microbunching (SIM) exhibited clear spectral effects at the fundamental and second harmonic of the FEL wavelength. The $z$-dependent gain of the COTR was comparable to that of the SASE as expected. At the International FEL Conference in 2002, the authors of Ref. [5] reported localized spatial enhancements of the COTR images, and in fact reported two 250- $\mu \mathrm{m}$ FWHM clusters $120-\mu \mathrm{m}$ apart and different in wavelength by $2.9 \mathrm{~nm}$ in images taken after undulator \#6 of a string of nine as shown in Fig. 1. At that time it was proposed that there must be localized structures in the electron beam distribution generated perhaps in the chicane that the SASE process preferentially selected. Originally, the investigators checked the e-beam emittance with YAG:Ce screens located just after the chicane. Some intensity variations of $10 \%-20 \%$ across the profile were seen, but it was not clear such fluctuations were enough to be selected preferentially by the SASE process. (The YAG: Ce scintillation strength would have masked the COTR effects to a large extent.)

As it was understood at that time, there was no theory that supported the coherent synchrotron radiation (CSR) microbunching instability's extending to visible wave- lengths in the linac [6,7]. Subsequently, it was proposed that the longitudinal space charge (LSC) fields might be the main effect in driving the microbunching instability at FIR wavelengths in a linac $[8,9]$. We now propose that the LSCCSR microbunching instability in the linac at 300-A peak current had effected the observed spatially localized SASE-induced microbunching and related SASE effects in the visible light regime in those 2001 experiments at APS. We report the new evidence for this connection. We have reviewed the archived data taken at an optical tran-

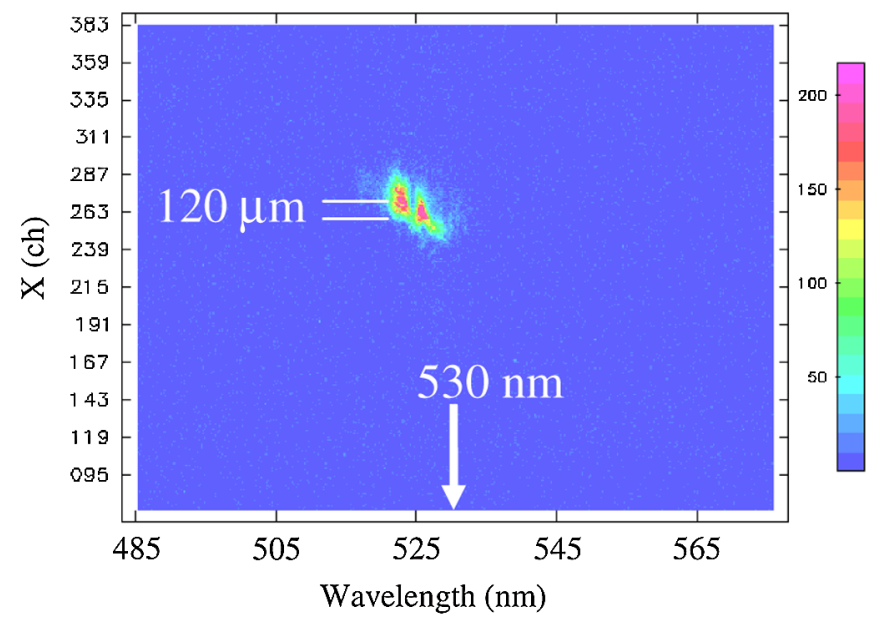

FIG. 1. (Color) An example of the imaging spectrometer data after undulator 6 showing the COTR $x$ - $\lambda$ dependence at $\sim 530 \mathrm{~nm}$ first reported at FEL02 [5]. 
sition radiation (OTR) screen just before and after the first undulator, and strong, spatially localized enhancements are clear in the 300-A data. These are comparable to the Fig. 1 spatial structures seen after undulator 6. More recently, "unexpected" enhancements of the signals in the visible light-IR OTR monitors (CCD cameras) were observed after compression in a chicane bunch compressor at Linac Coherent Light Source (LCLS) [10] and APS [11].

\section{EXPERIMENTAL BACKGROUND}

The measurements were performed at the APS facility which includes an injector complex with two thermionic cathode (TC) rf guns for injecting an $S$-band linac that typically accelerates the beam to $325 \mathrm{MeV}$, the particle accumulator ring (PAR), the booster synchrotron that ramps the energy from 0.325 to $7 \mathrm{GeV}$ in $220 \mathrm{~ms}$, a booster-to-storage-ring transport line, and the $7-\mathrm{GeV}$ storage ring (SR). In addition, there was a photocathode (PC) rf gun that could also be used to inject into the linac and ultimately to provide beam to the FEL as shown schematically in Fig. 2. An extensive diagnostics suite is still available in the chicane and after the chicane area as described in Ref. [11]. The microbunching instability tests were performed in the linac at the three imaging stations after the chicane bunch compressor and at the end of the linac where another beam imaging station is located. A FIR coherent transition radiation (CTR) detector (Golay cell) and Michelson interferometer are located just before the third screen of the three-screen emittance stations. This system is described in more detail elsewhere [12].

The YAG:Ce emissions and OTR were directed by turning mirrors and relay optics to a Pulnix CCD camera located $0.5 \mathrm{~m}$ from the source. These chicane stations also have options for low- and high-resolution imaging of the beam spot by selecting one of two lens configurations [13]. The near-field, low-resolution magnification resulted in calibration factors of $48.8 \mu \mathrm{m}$ per pixel in $x$ and $38.9 \mu \mathrm{m}$ per pixel in $y$. The OTR signal strength with a 400-pC micropulse was too low to use the high-resolution mode. The signal intensity was adjusted with a remotely controlled iris (with no absolute position readback) in the path to the camera. The OTR and YAG:Ce images were recorded by a Datacube MV200 video digitizer for both online and offline image analyses, and a video switcher was used to select the camera signal for digitizing.

At the end of the linac, the imaging station included the optical transport of the visible light out of the tunnel to a small, accessible optics lab where the Vicon CCD camera was located. This allowed the access for manually inserting bandpass (BP) filters and neutral density (ND) filters in front of the camera to assess the spectral dependency of the enhanced OTR. A set of BP filters with center wavelengths in 50-nm increments from 400 to $750 \mathrm{~nm}$ and $40-\mathrm{nm}$ bandwidth as well as a 500-nm shortpass (SP) filter and 500-nm long pass (LP) filter were used in the initial tests [11]. However, recently the Oriel spectrometer and readout cameras were moved from the FEL hall to this station to extend our spectral analysis capabilities for the LSCinduced microbunching (LSCIM) COTR effects. The beam energy was $325-375 \mathrm{MeV}$ at this station in these recent experiments.

The YAG:Ce (YN; $N=0-9$ ) stations and the visible light diagnostics (VLDN; $N=0-9$ ) stations in the FEL are schematically shown in pairs in Fig. 2 with a detailed view in Fig. 3. They were designed to assess the $z$-dependent SASE evolution with both near-field and far-field imaging optics and to track the e-beam position and size through the undulator string. They included the filter wheels that al-

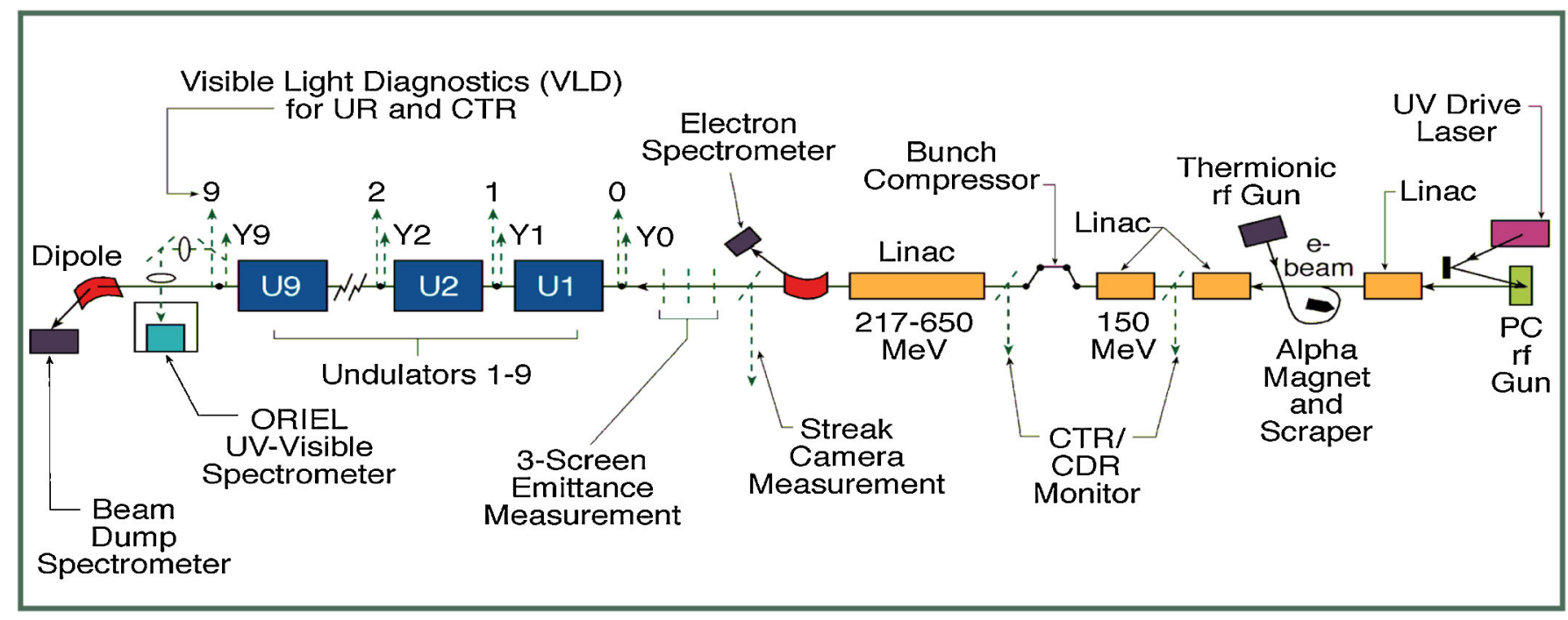

FIG. 2. (Color) A schematic of the APS linac test area showing the PC rf gun, the TC rf gun, the accelerator structures, the chicane, the decommissioned FEL with VLD stations after each of nine undulators, the imaging optical spectrometer, and the electron beam dump circa 2001 (Ref. [5]). 


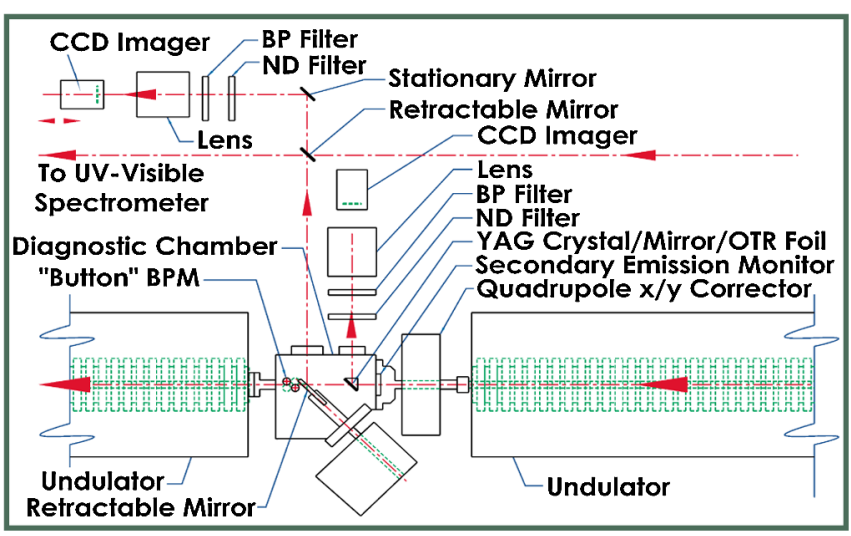

FIG. 3. (Color) A schematic of the YN and VLDN stations located before the first undulator and after each of the nine undulators at the time of the SASE-induced COTR experiments.

lowed the remote selection of ND filters and BP filters for insertion in the radiation path to the cameras. They were augmented to provide an OTR/blocking foil at the first YN actuator. This foil was used to prevent the SASE and spontaneous emission radiation from reaching the second, retractable pickoff mirror oriented at 45 degrees to the beam direction and $63 \mathrm{~mm}$ downstream of the first actuator. This configuration was used in the first SIM COTR experiments in the visible regime $[2,3]$ at this downstream portion of the station. The data being reassessed are from a comprehensive set of SIM tests at two peak currents, 120 and $300 \mathrm{~A}$, taken on December 20, 2001. The VLD0 station is before the first undulator and provides the key OTR images at the two currents. Evidently, the threshold for seeing the LSCIM COTR effects in the visible regime was passed at the higher peak current with 2.5 times more compression.

\section{RECENT CTR AND COTR RESULTS}

The more recent experiments [11] were initiated by transporting the PC gun beam accelerated to $150 \mathrm{MeV}$ to the chicane area. The rf phase of the L2 accelerator structure located before the chicane was used to establish the appropriate conditions for compression in the chicane. The degree of compression was tracked with the Golay cell signals. A very strong variation of the FIR signal with L2 phase was observed as shown in Fig. 4. There were only a few units of CTR signal seen with the beam uncompressed and 300 units seen at the peak compression. The autocorrelation scan was then done and showed a profile width of $\sim 65 \mu \mathrm{m}$ (FWHM). This would mean a round-trip time of $130 \mu \mathrm{m}$, or about $430 \mathrm{fs}$ (FWHM). The initial UV drive laser pulse was 3 to 4 ps (FWHM) as determined by an autocorrelator.

Having determined we had maximum compression from the Golay cell data, we then sampled the beam images at the three screens after the chicane in the emittance mea-

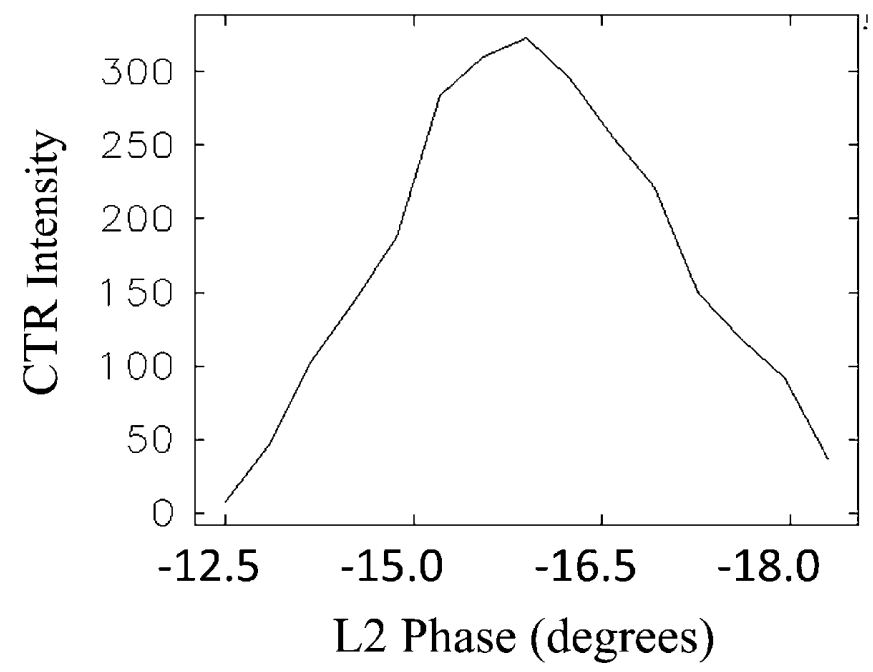

FIG. 4. FIR CTR signal from the Golay cell variation with the upstream L2 phase in degrees.

surement area. The sample shown is from FS5, the third screen of the set. At the point of minimum compression the OTR image is dim, and visibly tilted in $x-y$ space. In contrast, the image taken near full compression (as indicated by the FIR CTR signal) has significantly enhanced localized spikes of about $250 \mu \mathrm{m}$ (FWHM) spatial extent as shown in Fig. 5. The pseudocolor intensity scale at the right of the image shows that the red areas are high intensity. The profile in Fig. 5(b) shows the peak intensity of 180 counts, almost 10 times the adjacent intensities in the beam-image footprint. We do not see the ringlike structure in the enhancement reported at LCLS [10], but we also do not have a harmonic cavity for linearizing longitudinal phase space before the chicane as LCLS has. On this particular run, we noted that there was a preferred location in the footprint to be enhanced. The enhanced vertical band in Fig. 5(a) is the same area that shows less enhancement with less compression.

An additional indication of the effect is shown in Fig. 6, where the peak intensities from the vertical profiles taken through the images at each phase setting show the rapid increase in the enhanced OTR signal in just a few degrees of L2 phase change. The order of magnitude enhancement is clearly seen in this plot. A similar plot for horizontal profiles was done, and this showed a slight shift of the intensity peak compared to the CTR data by 0.4 degrees in L2 phase. Integrated areas of the ten images at each phase setting do not show this degree of enhancement, but about a 1.6 to 2 times larger counts integral at peak compression was seen with larger fluctuations in the compressed conditions. This fact is consistent with the premise that neither the charge transport efficiency nor CSR-induced clumping of electrons is the cause of the effect, but rather a coherent enhancement of some kind is involved.

In order to assess the spectral dependency of the OTR enhancements, we accelerated the beam to $375 \mathrm{MeV}$ and 

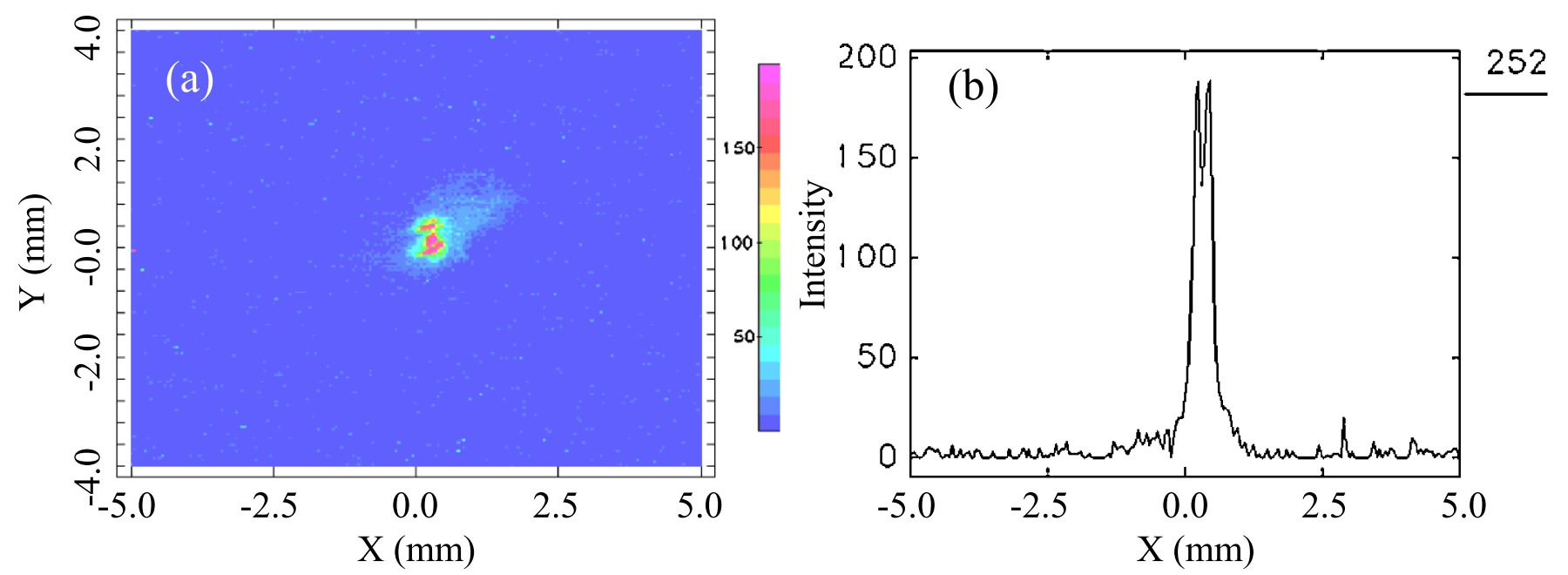

FIG. 5. (Color) OTR beam image (a) and horizontal profile (b) through spike in distribution after the chicane with L2 phase $=$ 14.9 degrees, close to maximum compression [11].

again imaged the beam spot with OTR at a downstream station. As described previously, this station included transport of the signal outside of the tunnel to a small optics lab. First, we still see enhanced localized spikes when we have compressed the beam as shown previously [11]. In this case enhancements of the OTR signals when at maximum compression are about 3-6 times the normal intensity. The strong fluctuations of these enhanced areas are again suggestive of a coherent process seeded by noise.

At full compression we checked the spectral dependency of the enhancements by inserting the BP filters in front of the CCD camera. Our initial results are that the enhancements were seen at all central wavelengths from 400 to $750 \mathrm{~nm}$ (in steps of $50 \mathrm{~nm}$ ), although much weaker in the 400 - to $500-\mathrm{nm}$ regime than at $550 \mathrm{~nm}$. We subsequently checked the spectral dependence of incoherent OTR from the TC gun beam and saw an intensity roll off in this

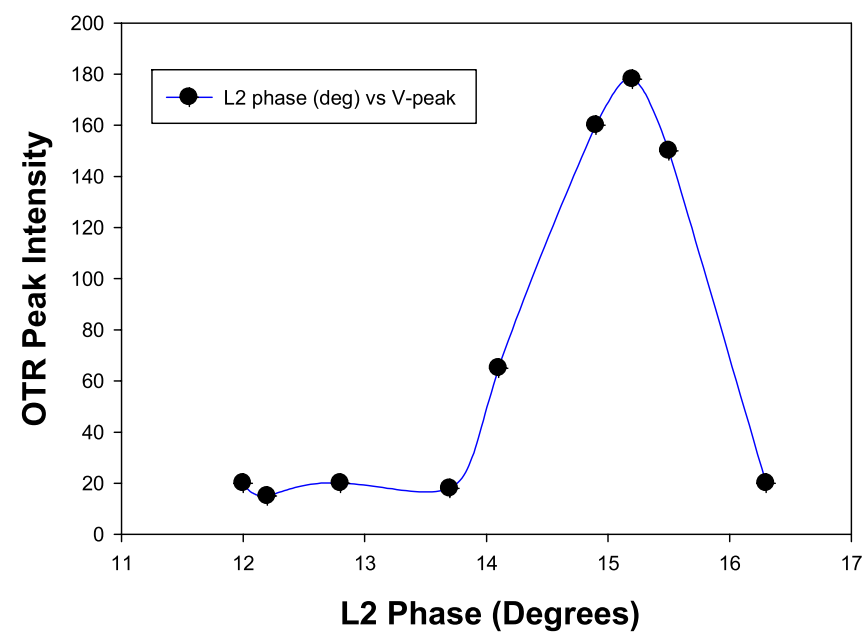

FIG. 6. (Color) Plot of the peak intensities from the OTR image vertical profiles taken near the enhanced regime coordinates as a function of L2 phase in degrees [11].
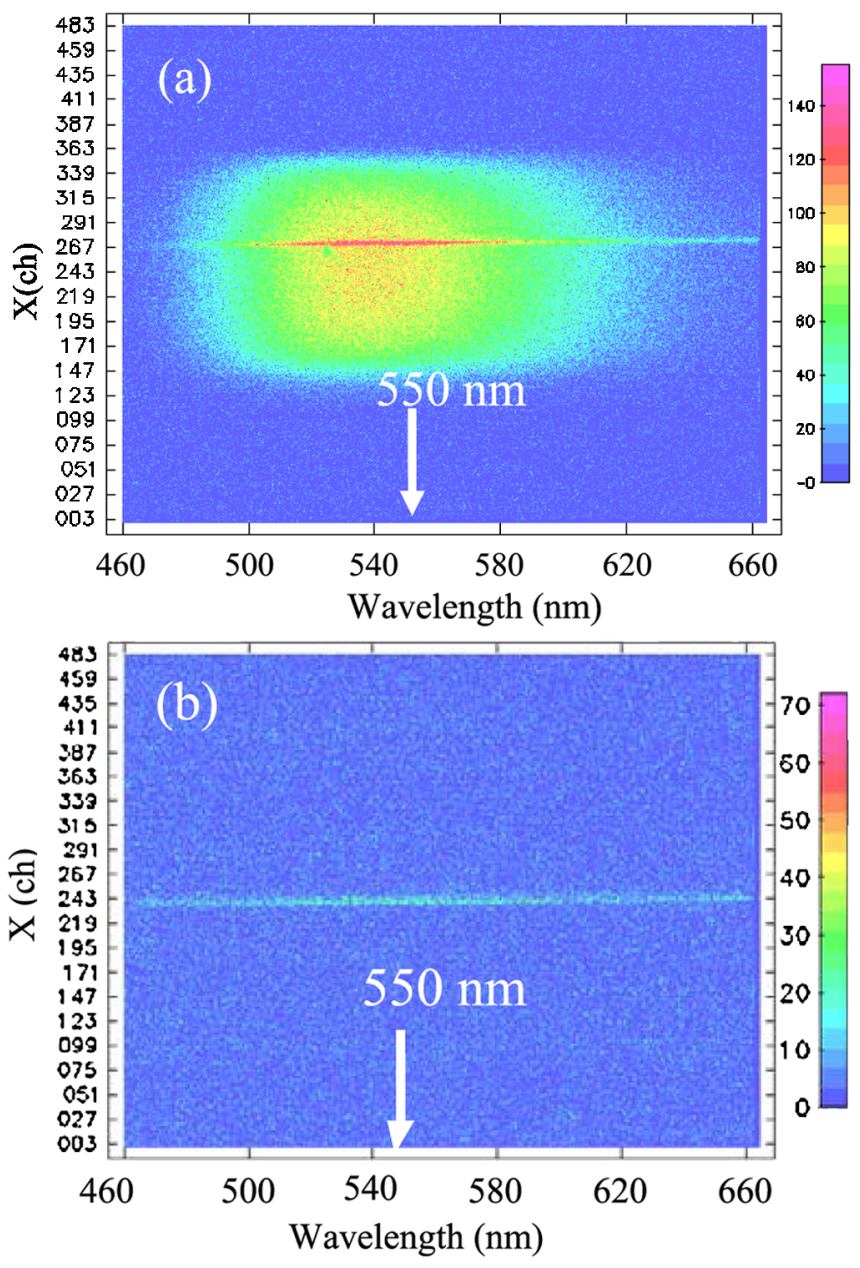

FIG. 7. (Color) Example $x$ - $\lambda$ images of the present PC gun beam at $375 \mathrm{MeV}$ near maximum bunch compression for (a) YAG:Ce screen plus "COTR streak" and (b) the COTR screen only. In this case the 95 lines/mm grating was selected in the spectrometer, and the wavelength span is about $180 \mathrm{~nm}$. The broadband emission streak is clearly from a localized $x$ location in both images. 
shorter wavelength interval which we can attribute partly to the standard manufacturer's CCD camera response curves for these different wavelengths [14]. However, this rolloff was not as much as the observed COTR intensity rolloff.

Additionally, in Fig. 7 we show our results from the spectrometer when positioned at a station at the end of the linac. The YAG:Ce light in Fig. 7(a) peaks at about $530 \mathrm{~nm}$ and shows the full spatial $x$ size of the beam as about $5 \mathrm{~mm}$ in the vertical display axis. The broadband spectral streaks originate from localized $x$ structures (sub-200 $\mu \mathrm{m}$ ) which appear with bunch compression in both cases. The YAG:Ce screen setup uses an $\mathrm{Al}$ mirror at 45 degrees to the beam to direct the light to the camera. This mirror is in the same plane as the OTR converter mirror when it is inserted so we can detect COTR from it as well. The spectrometer entrance slit was opened to $2000 \mu \mathrm{m}$ to facilitate the first data acquisition so the spectral resolution was broadened to about $40 \mathrm{~nm}$. In subsequent experiments with an ICCD readout camera, we were able to narrow the entrance slit and obtain few-nm resolution $[15,16]$.

\section{PAST OTR, COTR, AND SASE RESULTS WITH PC RF GUN BEAMS}

The purpose of this section is to introduce experimental evidence for enhanced OTR attributable to LSC-induced microbunching. We are now able to connect our December 2007 LSCIM COTR data with an original series of experiments that were performed on December 20, 2001. The objectives then were to explore the SASEinduced microbunching in the FEL as probed with COTR-based techniques using near-field focusing. In addition, we used a 500-nm shortpass filter to exclude the $530-\mathrm{nm}$ fundamental SASE or COTR in the low gain region to check the incoherent broadband image. The beam energy was $217 \mathrm{MeV}$. Now this filter also lets us look for enhancements that are not SASE induced. In this particular run we only had one filter available with a factor of about 100 rejection ratio for wavelengths longer than $500 \mathrm{~nm}$, so we cannot rely on this to completely reject the SASE-induced contributions after several gain lengths along $z$. We took data at both 120 and 300 A peak current.

TABLE I. Summary of the beam parameters for uncompressed and compressed states in the previous COTR and SASE tests at $530 \mathrm{~nm}$ performed on December 20, 2001 [5].

\begin{tabular}{lcc}
\hline \hline Parameter & Uncompressed & Compressed \\
\hline Beam energy $(\mathrm{MeV})$ & $217 \mathrm{MeV}$ & 217 \\
Emittance $x(\mathrm{~mm} \mathrm{mrad})$ & 4.6 after chicane & 4.1 after chicane \\
Emittance $y(\mathrm{~mm} \mathrm{mrad})$ & 4.3 after chicane & 4.2 after chicane \\
Peak current $(\mathrm{A})$ & 120 & 300 \\
Bunch length (ps rms) & 1.6 & 0.65 \\
Charge (pC) & 450 & 450 \\
Energy spread $(\%)$ & $0.1-0.2$ & $<0.1$ \\
\hline \hline
\end{tabular}

The beam parameters are shown in Table I [5]. The comprehensive test series also included $z$-dependent gain measurements of both COTR and SASE, including the critical VLD0 reference data taken just before the first undulator. In Fig. 8 we show the beam image from VLD0 at $120 \mathrm{~A}$. It is a relatively weak OTR image with $450 \mathrm{pC}$ of charge. This is compared to a sample image from VLD0 in the 300A run which clearly has localized spatial enhancements in it as shown in Fig. 9. The same color intensity scale is used, and we added ND 0.5 in the 300-A case to avoid camera saturation. The peaks of Fig. 9 are thus 5-6 times stronger than the 120-A case peak in Fig. 8. The 100-image integrated intensity averages were 2 times stronger in the 300A case than the 120-A case for very similar micropulse charge. These results are clear evidence for a coherent enhancement of some kind which we now attribute to the LSC microbunching instability and subsequent visible light COTR emissions. (We note that we have recently recommissioned the VLD0 station, transported compressed PC-gun beam to this station, and again observed
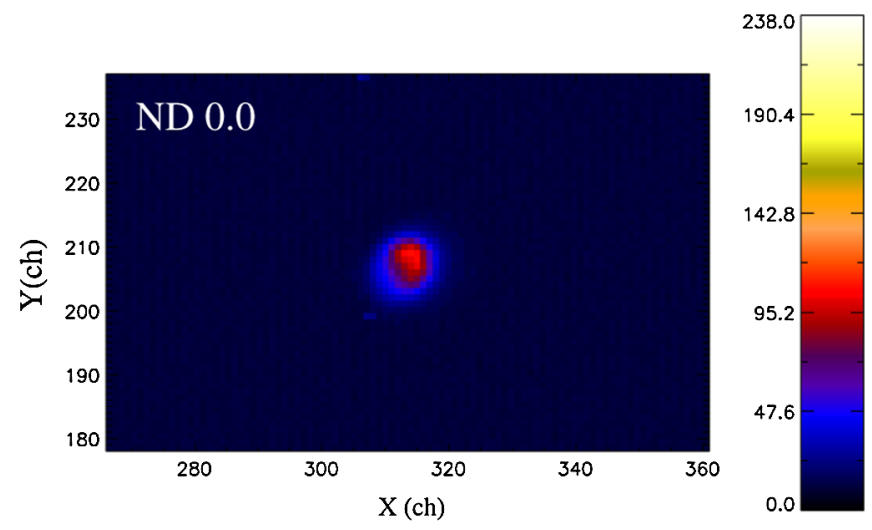

FIG. 8. (Color) An example of VLD0 OTR data with the 120-A beam and 450-pC micropulse with ND 0.0 .
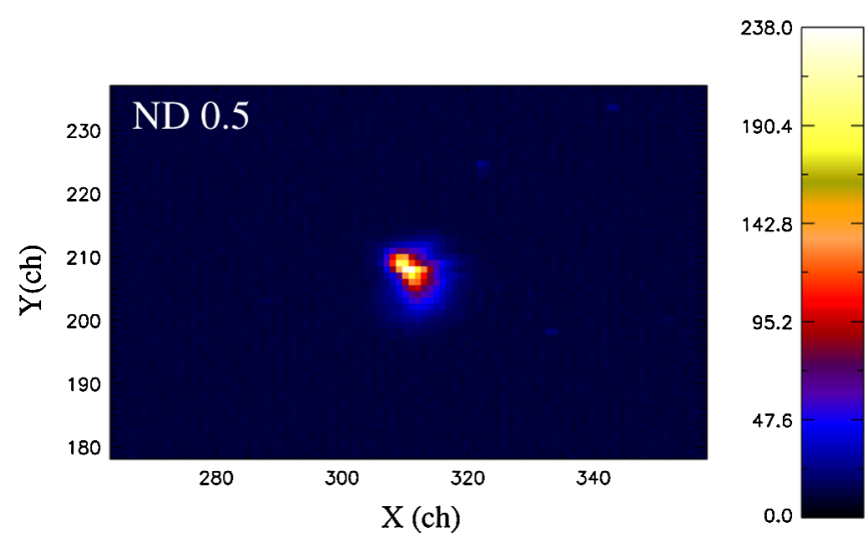

FIG. 9. (Color) An example of VLD0 OTR/COTR data with the 300-A beam and 450-pC micropulse with ND 0.5. The enhanced peaks are about $440 \mu \mathrm{m}$ apart. 

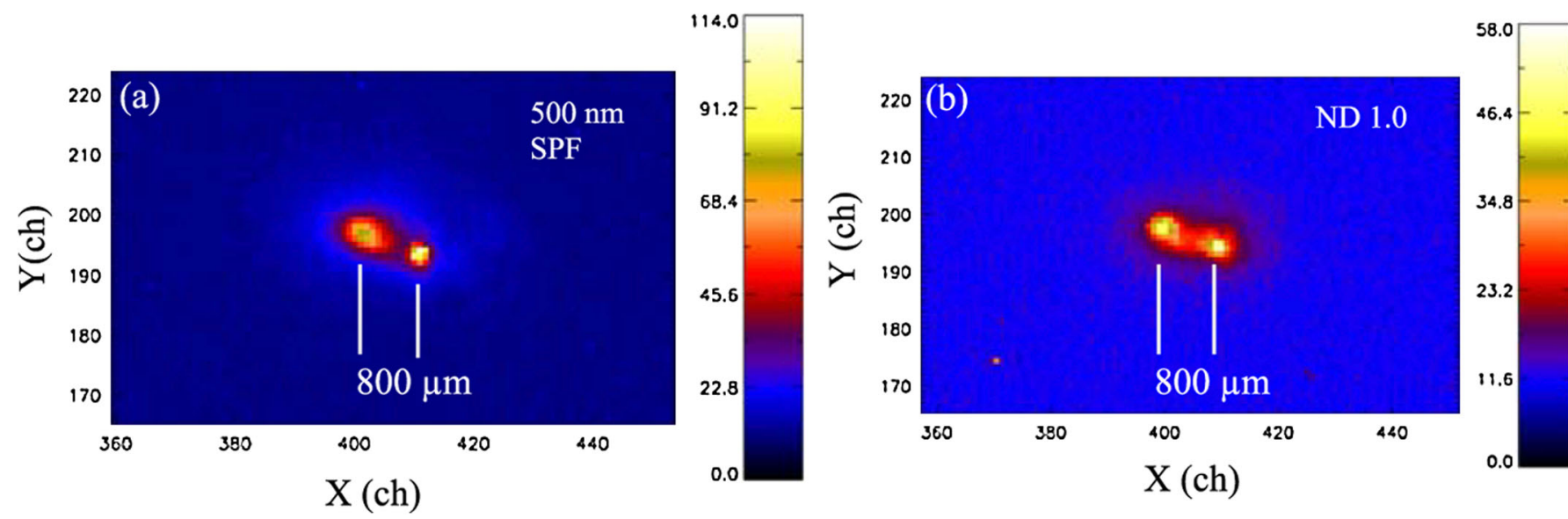

FIG. 10. (Color) Two examples of VLD1 OTR/COTR data with the 300-A beam for a 450-pC micropulse: (a) The image with 500-nm shortpass filter that blocks 530-nm SASE-related COTR. (There are two distinct peaks shown attributed to LSCIM) and (b) The SIM + LSCIM COTR image with ND1.0 filter and no shortpass filter. One sees a similar two peaks, but with about 5 times more intensity.

L2 phase effects on the observed COTR intensity. This test clearly links the two experimental eras.)

We then checked that after one undulator the enhancements persist in the 500-nm SP filter data from VLD1 as shown in Fig. 10(a). Of course, we cannot take simultaneous data sets so this is a different beam micropulse, but the effect remains. We note the two localized spatial structures that are $800 \mu \mathrm{m}$ apart. The intensity scale on the right provides the factor of 5 ratio of the peak-to-base intensity. In addition, in Fig. 10(b) we show the SIM + LSCIM COTR image taken with a single ND1.0 filter whose peaks are thus about 5 times stronger than those of filtered LSCIM-only images. The localized spatial structures in the LSCIM image are enhanced in intensity in the SIM COTR images, and this is particularly clear in the VLD2 image profiles shown in Fig. 11. The two peaks $800 \mu \mathrm{m}$ apart are still apparent in Fig. 11(a) with the SP filter, although the right-hand peak is seen as $\sim 3$ times stronger than the left-hand peak now. However, in Fig. 11(b) with an ND 2.0 filter and no SP filter, the right-hand peak is now $\sim 100$ times brighter (SIM COTR mostly) than the shortpass-filtered data, and the left-hand peak is in the noise. The COTR peak is only $320 \mu \mathrm{m}$ (FWHM) which means only a part of the electron beam is involved in the SIM. Again such examples are representative of the 100 images taken in each configuration. This is strong support for our hypothesis that the SASE startup is being favored at localized LSCIM structures.

For comparison and perspective purposes, the $z$-dependent gain curves for the COTR and SASE for the 300-A cases are shown in Figs. 12 and 13, respectively, and compared to GENESIS [17] simulations. The base GENESIS calculations used the 300-A parameter set from Table I [18]. The COTR data clearly have a faster startup in the VLD1-2 region than the GENESIS curve and saturate after undulator 4 at $z \sim 10 \mathrm{~m}$ in Fig. 12. Since there was an $\mathrm{rf}$
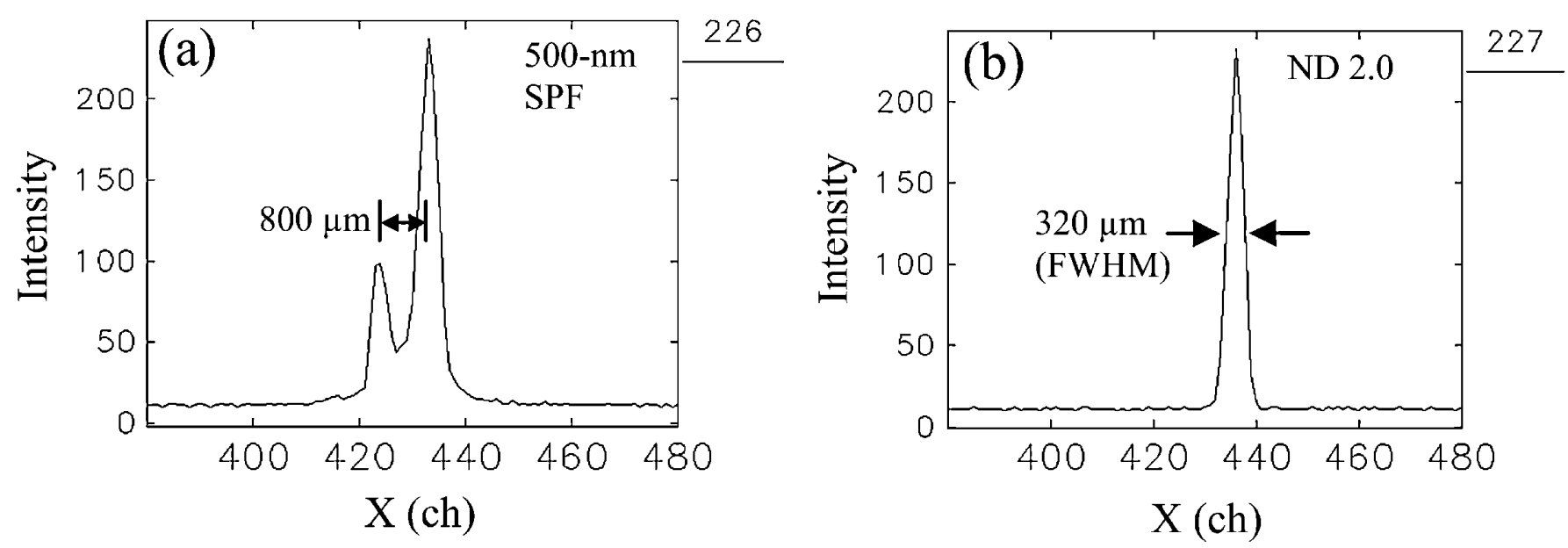

FIG. 11. Horizontal profiles from the 300-A VLD2 images (a) with the $500 \mathrm{~nm}$ shortpass filter (SPF) and (b) with an ND2.0 filter. The right-hand peak in (b) is enhanced by about 100 by SIM COTR. 


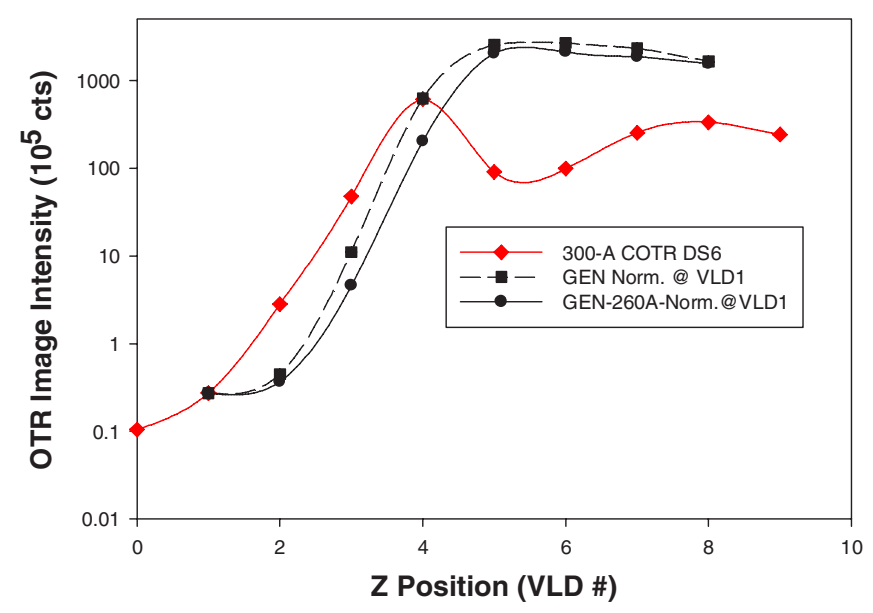

FIG. 12. (Color) The $z$-dependent COTR gain curve for SASEinduced microbunching at the FEL fundamental wavelength of $530 \mathrm{~nm}$ on December 20, 2001 (diamonds) and for recent GENESIS simulations of the microbunching fraction squared for the $300-\mathrm{A}$ case (squares) and the 260-A case (circles).

power trip in the linac during the $z$ scan after the VLD4 data were taken and only $87 \%$ charge transport was regained for the VLD5-9 data, a second GENESIS run was done with the corresponding 260-A peak current. For these two cases, the GENESIS-calculated microbunching fraction squared (which should scale like SIM COTR) are plotted. The COTR data are the most direct measure of the microbunching evolution at the SASE fundamental wavelength since the foils sample at discrete $z$ locations. However, the saturated signal levels of both GENESIS runs remain above the experimental data by a factor of 5-10. There are three SASE gain curves shown in Fig. 13 which saturate at about undulator 5; one experimental curve with 100-image re-

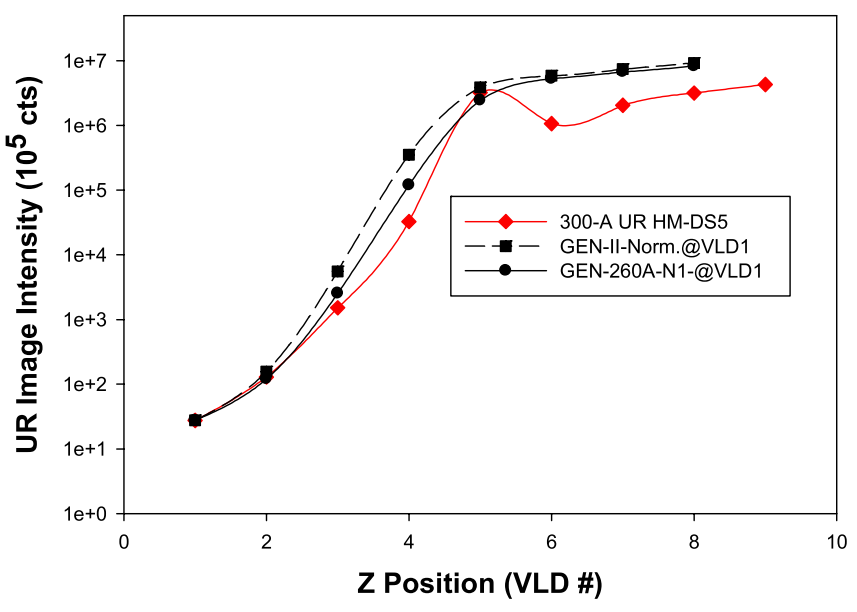

FIG. 13. (Color) The $z$-dependent gain curve for SASE at $530 \mathrm{~nm}$ taken by stepping through the nine VLD stations in decreasing order for data set 5 (diamonds) and for GENESIS simulations done for beam parameters for the 300-A case (squares) and the 260-A case (circles). gion of interest averages taken at each location for decreasing undulator number, and two from the GENESIS simulations recently done for 300-A and 260-A peak current [18]. In this figure the GENESIS curves were also normalized to the post undulator 1/VLD1 data location. The SASE experimental curve and GENESIS curves overlap up to VLD2, and then the simulation curves are seen above the data in the VLD3-4 area and overlapped again at VLD5. The combined COTR and SASE data-simulation comparisons are consistent with the hypothesis of extra microbunching contributions (LSCIM) beyond the SIM source as viewed through COTR and a concomitant localized startup effect.

\section{A. COTR and SASE: Imaging spectrometer results}

Having demonstrated that the localized structures are present after VLD0, VLD1, and VLD2 with 300-A beam, we still need to check that they are also present in the VLD6 data that complement the COTR-6 spectrometer data. However, at that time we only had one SP filter in each station, and it could not cleanly block the strongly enhanced COTR at the resonant wavelength. In Figs. 1 and 14 we show the $x-\lambda$ images, one published in Ref. [5] and one now just being reported. Both of these, as well as many other images taken at that time, show the localized spatial structures. The $x$ axis is on the vertical display axis. In Fig. 14 the two enhancements are $\sim 150 \mu \mathrm{m}$ apart in $x$ and $\sim 3 \mathrm{~nm}$ apart in wavelength near the nominal 530-nm SASE fundamental wavelength. These data were recorded after SASE saturation was attained, and some debunching of the SIM had occurred as shown in Fig. 12.

Further review of the old data sets led to the finding of the SASE $x$ - $\lambda$ data shown in Fig. 15. There is structure on the $x$ direction in the vertical display axis. Since physically

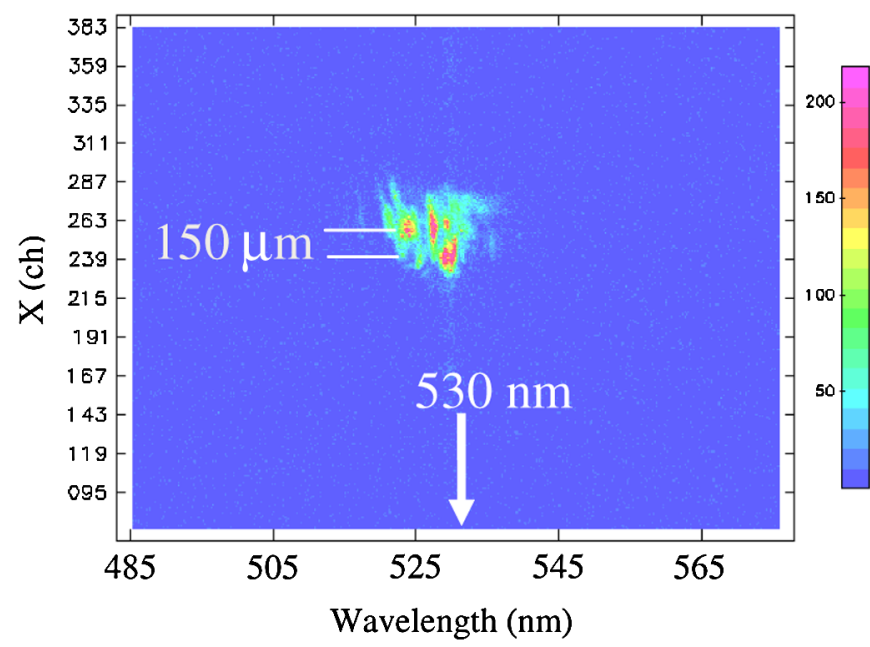

FIG. 14. (Color) Another example of the imaging spectrometer data showing the COTR $x$ - $\lambda$ dependence near the 530-nm fundamental wavelength after undulator 6 from December 20, 2001 experiments. 


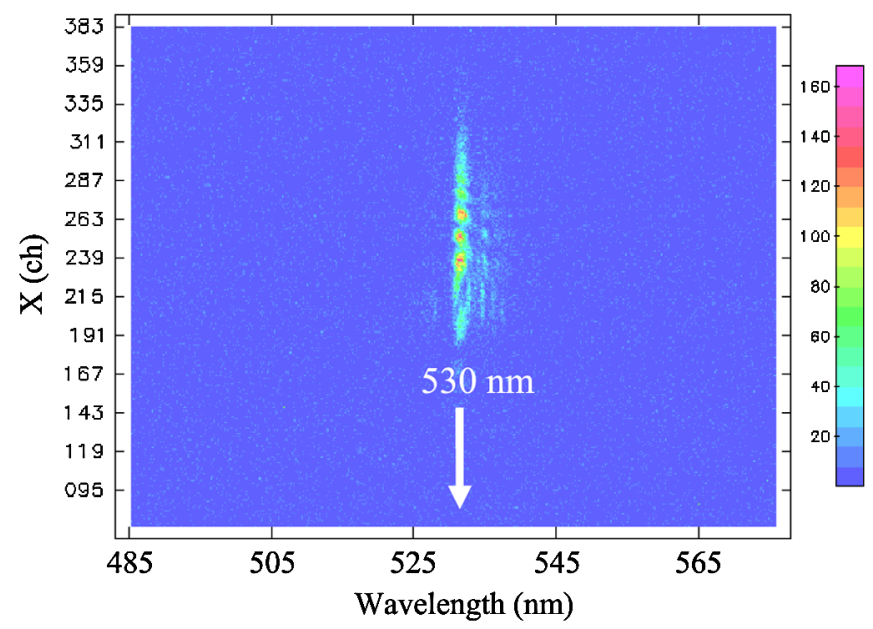

FIG. 15. (Color) An example imaging spectrometer image of undulator radiation sampled after undulator 6 with the 300-A beam from December 20, 2001. Localized spatial spikes are evident on the vertical display axis which is the $x$ spatial axis.

the spectrometer slit is along this direction, we do not believe this spatial intensity modulation is a diffraction effect from that particular slit. This feature needs additional review of other images and stations.

\section{B. Discussion}

We had identified several characteristics of the enhanced OTR signals that were used to evaluate which mechanism was involved [11]. If we consider charge transport efficiency, radiation enhancements, and spectral structure observed, we can discriminate among microbunching, a longitudinal spike with a sharp leading edge, multiple submicron spikes, and CSR-induced clumping. We initially considered whether there was microbunching from the drive laser interaction with the low-energy photoelectrons such as found under high laser power conditions, but a simple calculation of the laser field strengths showed this effect would not be measurable [11]. Since the transported charge through the chicane and undulators in the past was constant to $10 \%$ during the phase scan, we cannot explain the large, spatially localized OTR enhancements as only due to CSR-induced clumping of the charge distribution. Assuming the clumping does not involve microbunching, there would be no way to explain the observed OTR enhancements of the total image intensities by factors of two or more. Finally, our initial spectral sampling across the visible light region with 40-nm bandwidths showed a broadband character like that of incoherent OTR, but the COTR was relatively red enhanced [16]. Additionally, in Fig. 7 we show our initial results from the spectrometer (with CCD readout) when positioned at a station at the end of the linac. The broadband streaks clearly originate from the localized $x$ structures which appear with bunch compression. Such post-chicane data are consistent with co- herent enhancements of wavelengths longer than a fine longitudinal structure, such as a leading-edge spike. However, a recent report by Huang [19] also shows a broadband enhancement in the visible regime due to the LSC instability and the chicane compression process. We suggest another discriminating test is whether LSCinduced microbunching would act as "spatially localized prebunched beam" and induce startup of visible wavelength SASE FELs at these structures, and that aspect is what was actually reported six years ago in the FEL02 transverse-dependencies paper [5] and is reconfirmed in Figs. 8-14.

\section{SUMMARY}

In summary, we have connected for the first time the previously reported transverse dependencies on SIM COTR reported at FEL02 with broadband LSCIM effects as identified in investigations described over the past year. This key identification was based on a review and interpretation of the 300-A data taken at ANL in 2001. Common to these two sets of ANL data are the drive laser, photocathode material, the linac, the chicane, and the optical spectrometer. The present PC rf gun beam's transverse emittance is larger than in 2001 ( $\sim 10$ versus $4 \pi \mathrm{mm} \mathrm{mrad}$ ), but it has comparable longitudinal emittance. We will be reviewing other data sets from past ANL visible, UV, and VUV SASE experiments as time permits. Start-to-end simulations would be welcomed that investigate this proposed connection of LSCIM and SASE startup. The growing interest in these COTR effects is indicated by the time allowed for discussion in the recent high-brightness beams workshop at Zeuthen in May 2008 [20] and the Microbunching Instability Workshop II in October 2008 at Berkeley [21]. It remains to be confirmed if this LSC microbunching instability played, or will play, a role in FEL startup in the optical and infrared regime for other experiments.

\section{ACKNOWLEDGMENTS}

The authors acknowledge support from M. Wendt of Fermilab and R. Gerig, K.-J. Kim, and H. Weerts of the Argonne Accelerator Institute. We acknowledge Y.-C. Chae for providing the new GENESIS simulations. We acknowledge discussions with K.-J. Kim, Y.-C. Chae, and M. Borland of Argonne National Laboratory; Z. Huang of SLAC; A. Gover of the University of Tel Aviv; and W. Fawley of LBNL. They also acknowledge W. Berg and S. Shoaf of Argonne National Laboratory for the recent relocation of the optical spectrometer and cameras with controls to the small diagnostics lab linked to linac Sta-5. This work was supported by the U.S. Department of Energy, Office of Science, Office of High Energy Physics, under Contract No. DE-AC02-06CH1135. 
[1] S. V. Milton et al., Science 292, 2037 (2001).

[2] A. H. Lumpkin et al., Phys. Rev. Lett. 86, 79 (2001).

[3] A.H. Lumpkin et al., Phys. Rev. Lett. 88, 234801 (2002).

[4] A. H. Lumpkin et al., Nucl. Instrum. Methods Phys. Res., Sect. A 483, 402 (2002).

[5] A.H. Lumpkin et al., "Evidence for Transverse Dependencies in COTR and Microbunching in a SASE FEL," in Proceedings of the 23rd FEL Conference, Argonne, IL, 2002; Nucl. Instrum. Methods Phys. Res., Sect. A 507, 200 (2003).

[6] M. Borland et al., Nucl. Instrum. Methods Phys. Res., Sect. A 483, 268 (2002).

[7] E. L. Saldin, E. A. Schneidmiller, and M. V. Yurkov, Nucl. Instrum. Methods Phys. Res., Sect. A 490, 1 (2002).

[8] E. L. Saldin, E. A. Schneidmiller, and M. V. Yurkov, DESY Report No. TESLA-FEL-2003-02, 2003.

[9] Z. Huang et al., Phys. Rev. ST Accel. Beams 7, 074401 (2004).

[10] D.H. Dowell et al., in Proceedings of FEL07, Novosibirsk, 2007, WEAAU01.

[11] A. H. Lumpkin et al., "Observations of Enhanced OTR Signals from a Compressed Electron Beam," in
Proceedings of BIW08, Tahoe, CA, 2008 (to be published).

[12] A. H. Lumpkin et al., in Proceedings of FELO5 eConf C0508213, 608 (2005).

[13] B. Yang, E. Rotela, S. Kim, R. Lill, and S. Sharma, AIP Conf. Proc. 648, 393 (2002).

[14] For example, see the CCD sensitivity charts which include Pulnix cameras on http://www.jai.com.

[15] A. H. Lumpkin et al., "Spectral and Charge-dependence Aspects of Enhanced OTR Signals from a Compressed Electron Beam,' in Proceedings of Linac08, Victoria, BC, 2008 (to be published).

[16] A. H. Lumpkin, N. S. Sereno, W. Berg, M. Borland, Y. Li, and S. Pasky (unpublished).

[17] S. Reiche, Nucl. Instrum. Methods Phys. Res., Sect. A 429, 243 (1999).

[18] Y.-C. Chae (private communication).

[19] Z. Huang et al.,in the Mini Workshop on Characterization of High Brightness Beams, Zeuthen, Germany (2008).

[20] Agenda for Mini Workshop on Characterization of High Brightness Beams, Zeuthen (2008).

[21] Agenda for Microbunching Instability Workshop II, Chairman W. M. Fawley, LBNL, Berkeley CA, 2008. 